\title{
Dark Matter Might Reside in Extra Physical Dimensions
}

\author{
Ahmad Yousef ${ }^{1}$ \\ ${ }^{1}$ School of Computational Science and Engineering, McMaster University, Ontario, Canada \\ *Correspondence: mohamas2@mcmaster.ca
}

A gravitational lens is a distribution of matter between a distant light source and observers, which has the ability to bend the light from the source as the light travels towards the observers. Humans/telescopes may experience simultaneous viewing of a certain galaxy in one or more different cosmic spaces, with different sizes and orientations due to gravitational lensing. When matter is invisible to all of our observatories, then dark matter is suspected to achieve gravitational lensing. In a significant cosmological event, NASA Chandra X-ray observatory telescope has focused its attention to collect influential information about a distance galaxy in the background of the bullet cluster (1E 0657-558) which consists of two colliding clusters of galaxies. Unexpected pictures are obtained after the end of the collision, namely, the position of the distance galaxy had been significantly changed with no evidence of residual matter in the collision site. The collision site might be therefore held dark matter, which is invisible to all of electromagnetic telescopes.

In his lecture, see reference 3, Brian Greene had suggested to examine whether there is extra dimension by a well-designed massive collision experiment in the large hadron collider. He believes that; if the total mass/energy before the collision is larger than the total mass/energy after the collision, then there must be some mass/energy ejected to extra physical dimensions. Greene's thought experiment about extra physical dimensions may be proven by rearranging the physical plausibility see references 4 , and 5 .

In case of the readers are not following our previous work, we here provide a simplified theoretical proof of the physical existence of tachyons according to the well-established theoretical physics: Imagine the Laser Interferometer Gravitational-Wave Observatory 'LIGO system' but with extremely short two tubes, namely, the tubes' length has to be the laser's wavelength; the LIGO detector will therefore detect a zero; and this seriously violates the energy conservation principle. To resolve the aforementioned violation; we suggested that the energy were conveyed to extra physical dimensions, but since the super-string theory assumes that these extra dimensions are too small; thus, the momentum of the particles travelling through these dimensions have to be way smaller than the photons. But we have to be attentive here, because if these particles' momentum have to be way smaller, then their speed must be way faster; otherwise, the energy conservation principle will be again violated, see the following energy equations for better illustration:

$E_{\text {Before destructive interference }}=E_{\text {After destructive interference }}$ $x=\operatorname{det}\left(P_{\text {photon }} \times C\right)=w=\operatorname{det}\left(P_{\text {tachyon }} \times Y\right)$

where the dot product of the vectors $x$, and $w \rightarrow 0 ; C$ and
$Y$ are the speeds of light, and tachyon respectively, and $P$ is the momentum; but the readers have to be again too careful that these are just mathematical illustrations; but our mathematical description for the tachyonic dynamics will be provided in a further notice. But it's very notable that the scientists had been searching for the "unknown" particles in incorrect manners; to understand the reasons, let's see the Planck's equation:

Energy $=$ Planc $k^{\prime}$ s constant $\times$ Frequency

But remember that because the LIGO detector shall detect a zero according to our thought experiment; therefore, the resultant particles will have zero value of the frequency; namely, the resultants' wavelength will be infinite. This suggests that scientists had been looking into the wrong planes and dimensions, namely, the Large Hadron Collider 'LHC system' will never be able to directly detect tachyons, unless their dynamics are considered in the detection design. The aforementioned explanation is to theorize that 'the collision of these two colliding clusters' triggered tremendous amounts of tachyonic transmissions out of collision site; and that dark matter is presumed to be in extra physical dimensions. Notice the tachyons, by definition, travel through extra physical dimensions but within certain 'gated tubes' that allow accesses to the $x^{2}-x^{2}$ a.s. designated dark matter. The tachyonic emissions, we assume, are to rearrange the distributions of the dark matter aiming to stabilize the entire system during and after the collision: and thus, the phenomenal gravitational lensing.

Similar to tachyons, because we assume that dark matter reside in extra dimensions which are orthogonal but linked to our spacetime continuum through 'gated tubes'; therefore, dark matter will remain undetectable to the conventional observatories until the detection design respects the tachyonic dynamics. But until scientists pay attention to the tachyonic dynamics, its mathematical description, and until detectors for tachyons are designed, see reference 5; no fulsome 'direct' findings will be reported in that regard. We're not trying to say that the indirect evidence of dark matter, and how they fit the observatories data are not convincing; but, if there is a gated vault in science, it's better to open it instead of speculating what's inside!

\section{Transactional References}

[1]" Invisible dark matter makes up most of the universe, but we can only detect it from its gravitational effects". CERN.

[2] "The Chandra X-ray Observatory: Overview". Chandra X-ray Center. 2015. [3] Brian Greene. (2008) " Making sense of string theory". YouTube, TED Channel, 2008. 16:14 to 19:06.

[4] Yousef, 2019. "Consciousness Might Be Localized in Extra Physical

Dimensions.” PsyArXiv. doi:10.31234/osf.io/angc8.

[5] Yousef, 2019. "Tachyons but Not Photons Might Generate Conscious Dreams." PsyArXiv. doi:10.31234/osf.io/hvqy7. 